\title{
Assessment of flow pattern of right ventricle outflow and pulmonary arteries in surgically corrected tetralogy of Fallot patients by four-dimensional cardiac magnetic resonance flow
}

\author{
Mahmoud Shaaban* ${ }^{*}$, Mai Salama, Ayman Alsaied, Raghda Elsheikh and Magdy Elmasry
}

\begin{abstract}
Background: The most common post-surgical complication of tetralogy of Fallot (TOF) is pulmonary regurgitation (PR) which can lead to right ventricle (RV) dysfunction/failure. Cardiac magnetic resonance (CMR) is the imaging modality of choice to follow-up a repaired TOF. However, the conventional two-dimensional phase-contrast (2D-PC) flow usually underestimates PR as well as the pulmonary peak systolic velocity (PSV). Recently, four-dimensional (4D) CMR flow is introduced for more accurate quantitative flow assessment. This work aimed to compare between 4D-CMR and 2D-PC flow across the main (MPA), right (RPA), and left (LPA) pulmonary arteries (PAs) in surgically corrected TOF patients.

Results: This study was conducted on 20 repaired TOF patients (range 3-9 years, 50\% males). All patients had CMR exam on 1.5T scanner. 4D-CMR and 2D-PC flows were obtained at the proximal segments of the MPA, RPA, and LPA. The stroke volume index (SVI), regurgitation fraction (RF), and PSV measured by 4D-CMR were compared to 2D-PC flow. The SVI across the PAs was nearly similar between both methods ( $P=0.179$ for MPA, 0.218 for RPA, and 0.091 for LPA). However, the RF was significantly higher by 4D-CMR in comparison to 2D-PC flow ( $P=0.027$ for MPA, 0.039 for RPA, and 0.046 for LPA). The PSV as well was significantly higher by 4D-CMR flow ( $P=0.003$ for MPA, $<0.001$ for RPA, and 0.002 for LPA). The Bland-Altman plots showed a good agreement between 4D-CMR and 2D-PC flow for the SVI, RF, and PSV across the pulmonary arteries.

Conclusion: A good agreement existed between the two studied methods regarding pulmonary flow measurements. Because of its major advantage of performing a comprehensive flow assessment in a shorter time, 4D-CMR flow plays an important role in the assessment of patients with complex CHD especially in the pediatric group.
\end{abstract}

Keywords: 4D-CMR flow, 2D-PC flow, TOF, Pulmonary regurgitation

\section{Background}

Tetralogy of Fallot (TOF) is one of the most common congenital heart disorder (CHD) and is the most common cyanotic CHD. Corrective surgery for TOF is usually performed in the first year of life [1]. It classically

* Correspondence: mahmoud.shaban@med.tanta.edu.eg

Cardiology Department, Faculty of Medicine, Tanta University, Tanta, Egypt consists of ventricular septal defect (VSD) closure and reconstruction of the right ventricle outflow tract (RVOT) [2]. RVOT reconstruction can be performed by trans-annular patch, RV to pulmonary artery (PA) conduit, or a valve-sparing technique. The most common post-surgical complication is pulmonary regurgitation (PR) which can lead to secondary RV volume overload $[3,4]$. 
The growing increment in endurance rate and the requirement for continuous clinical follow up are presently driving the need for improved cardiovascular imaging [1]. Cardiovascular magnetic resonance (CMR) is superior to echocardiography in the assessment of RV volume, quantification of $\mathrm{PR}$, and monitoring the progression of PR and RV dilatation [5, 6]. Additionally, the new timeresolved three-dimensional (3D) phase contrast CMR (the also called four-dimensional (4D) CMR flow) has been proposed for more accurate quantification of cardiovascular blood flow and for obtaining more detailed information about vascular hemodynamics [7]. Accordingly, this method may be a promising method to precisely assess altered blood flow in patients with CHD with complex anatomy and hemodynamics [8-10].

4D-CMR flow is particularly important given that characterization of intra-cardiac flow is much more challenging than flow assessment in vessels due to the 3D geometry of the cavity and changing dynamics during the heart cycle [11]. In particular, the RV has a more complex shape than the left ventricle (LV), which can complicate flow assessment.

The aim of this study was to assess the flow across the main pulmonary artery (MPA), as well as the right (RPA) and left (LPA) pulmonary arteries in surgically corrected TOF patients by $4 \mathrm{D}-\mathrm{CMR}$ flow and we compared the obtained results with the conventional twodimensional phase contrast (2D-PC) flow.

\section{Methods}

This study was conducted on 20 surgically repaired TOF patients who were presented for follow up at our institution in 2019. Eight patients were repaired with RVOT patch, 8 patients with trans-annular patch, and 4 patients with RV-PA conduit. CMR was performed on all included patients. A part of the 20 included patients, 8 patients were excluded from the study; 3 patients had atrial fibrillation affecting accurate flow measurements and the other 5 patients had poor CMR image quality. An informed written consent was obtained from care givers of all participants, and the study protocol was approved by the institutional ethics committee.

\section{Cardiac magnetic resonance imaging}

CMR examinations were performed on $1.5 \mathrm{~T}$ scanner (Siemens Magnetom Aera, Siemens Medical Systems, Erlangen, Germany) for all patients. The CMR exam included assessment of bi-ventricular volumes and systolic function, 2D-PC, and 4D-CMR flow across the MPA, RPA, and LPA.

\section{Bi-ventricular volumes and function}

Steady-state free precession (SSFP) Cine images were typically used to measure ventricular volumes and function. Images were acquired in three long axes planes; Cine 4-Chamber, 2-Chamber, and 3-Chamber by retrospective electrocardiogram (ECG) gating throughout the whole cardiac cycle to assess wall motion abnormalities and ventricular functions. Continuous stacks of SSFP Cine images were acquired in axial plane covering the entire ventricles to quantify ventricular end-diastolic (ED) and end-systolic (ES) volumes, stroke volumes, and ejection fractions.

\section{D phase contrast flow}

ECG triggered 2D-PC sequences were used for flow measurements and quantification of pulmonary regurgitation. The imaging planes were set perpendicular to flow in the main pulmonary artery as well as right and left pulmonary branches (Fig. 1). Flow data and the PR across the pulmonary vessels as well as the PSV across the MPA were measured using dedicated post processing software (Phillips intelliSpace Portal version 8.0).

\section{Four-dimensional CMR flow 4D-CMR flow data acquisition}

4D-CMR flow acquisitions were synchronized to the heart rate using prospective ECG-gating and adaptive diaphragm navigator gating. The CMR sequence consisted of a k-space segmented rf-spoiled gradient echo sequence with interleaved three-directional velocity encoding. 3D data volume was individually adapted to include the entire RVOT, MPA, RPA, and LPA (Fig. 2).

\section{D-CMR flow analysis}

Reconstruction of magnitude images for anatomy identification and flow data images was performed by a dedicated 4D-CMR post-processing software (Siemens 4D flow V 2.4).

\section{Qualitative flow analysis}

Qualitative visualization of flow pattern across the RVOT and the PAs was done. Traces along the measured velocities were color-coded ranging from blue to red color according to the local velocity in the different vessels of interest with the lowest velocity given the blue color and the highest velocity given the red color. Assessment of the vortex formation and angulation of the flow across the cardiac cycle was performed. Multiple approaches were used to visualize flow, including colorcoding mapping, vector fields, and streamlines (Fig. 3).

\section{Quantitative flow analysis}

Flow-time curves, PR, and PSV across the pulmonary arteries were analyzed through manually positioned planes in the MPA just above the pulmonary valve, and in the proximal segments of the RPA and LPA (Fig. 4). Stroke 


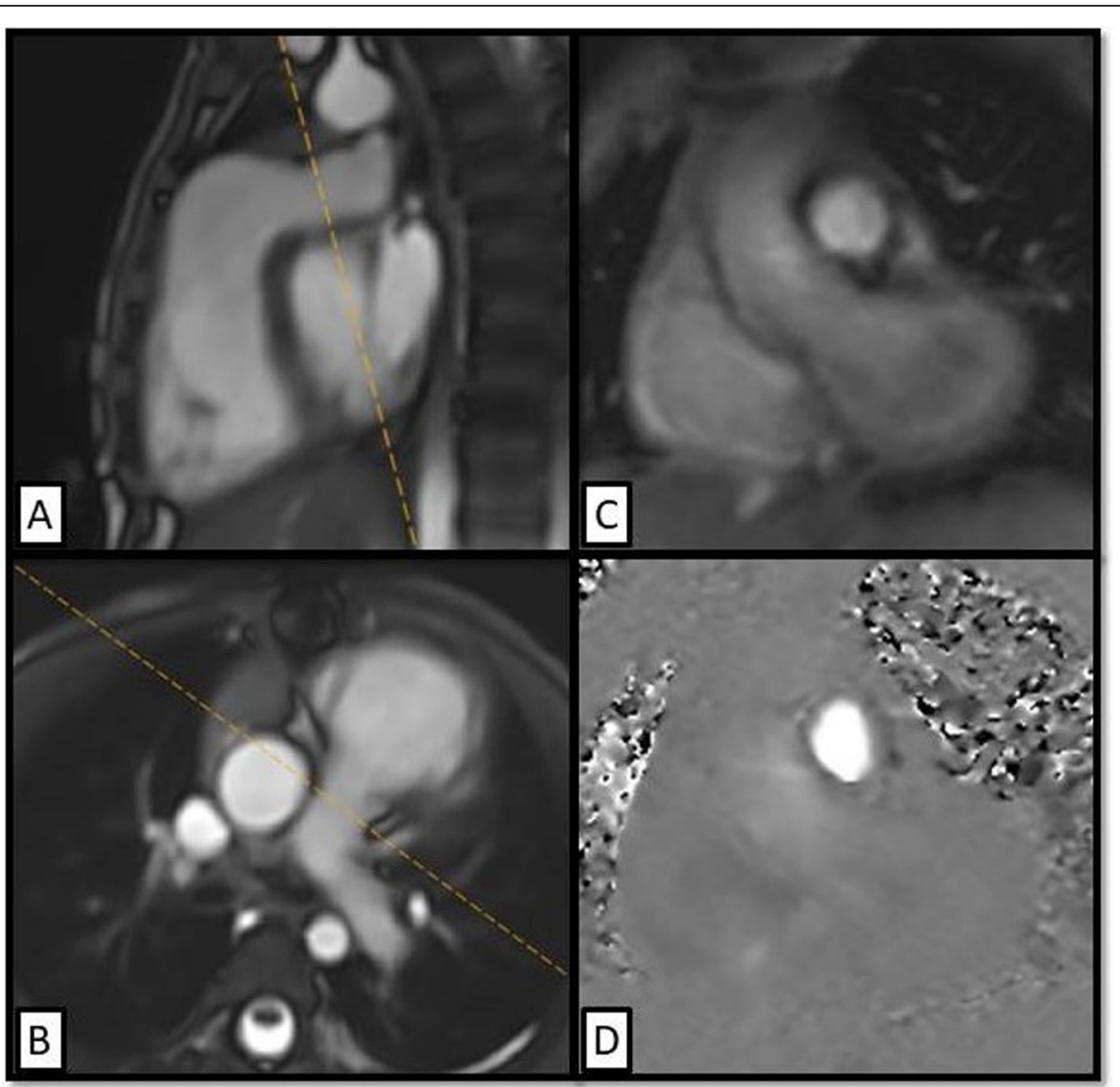

Fig. 1 Planning of 2D-PC flow across the MPA. $(\mathbf{a}, \mathbf{b})$ Two orthogonal planes taken on sagittal (a) and coronal (b) SSFP Cine images of the MPA. c The resultant anatomy magnitude cross section image of the MPA and $\mathbf{d}$ is the PC image showing the forward systolic flow across the MPA (white color). PC: phase contrast, CMR: cardiac magnetic resonance, MPA: main pulmonary artery, SSFP: steady-state free precession

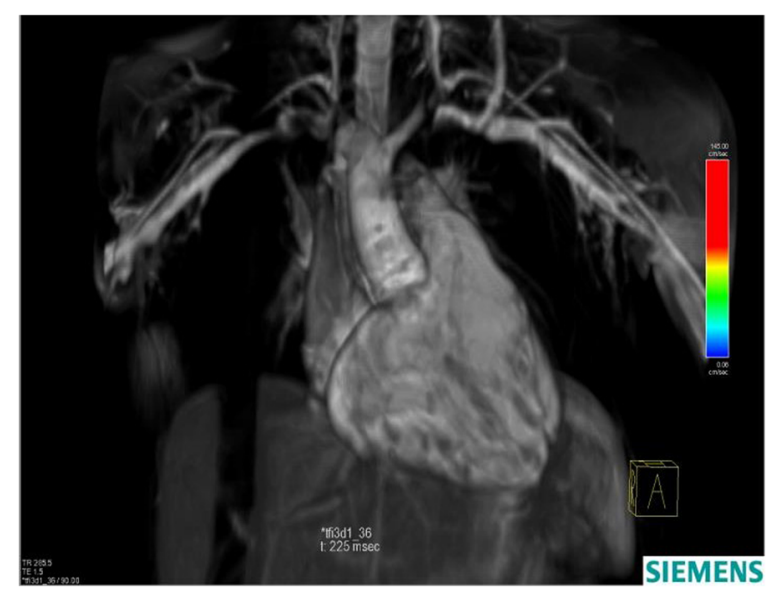

Fig. 2 3D data volume acquisition in a surgically repaired TOF patient with RVOT patch. TOF: tetralogy of Fallot, RVOT: right ventricle outflow tract volume (SV), RF, and PSV measured by 4D-CMR flow were compared to $2 \mathrm{D}-\mathrm{PC}$ measurements.

\section{Statistical analysis}

Statistical analysis was performed using IBM SPSS statistics 19.0.0 (SPSS Inc., Chicago, IL, USA). Nominal and continuous data were expressed as frequencies and mean \pm standard deviation as appropriate. Non-parametric statistical hypothesis test (the Wilcoxon signed-rank test) was used to compare results obtained by $4 \mathrm{D}-\mathrm{CMR}$ and 2D-PC flow. Bland-Altman plots were used to correlate between flow data obtained by $4 \mathrm{D}-\mathrm{CMR}$ flow and 2D-PC flow.

\section{Results}

Our study included 20 surgically repaired TOF patients (median age 4 years, range 3-9 years, 50\% males, mean body surface area was $0.686 \pm 0.143 \mathrm{~m}^{2}$ ). Eight patients (40\%) had surgical repair with RVOT patch, 8 patients (40\%) with trans-annular patch, and 4 patients $(20 \%)$ were repaired with RV-PA conduit. The mean follow-up period by CMR after surgical correction was $2.4 \pm 1.3$ 


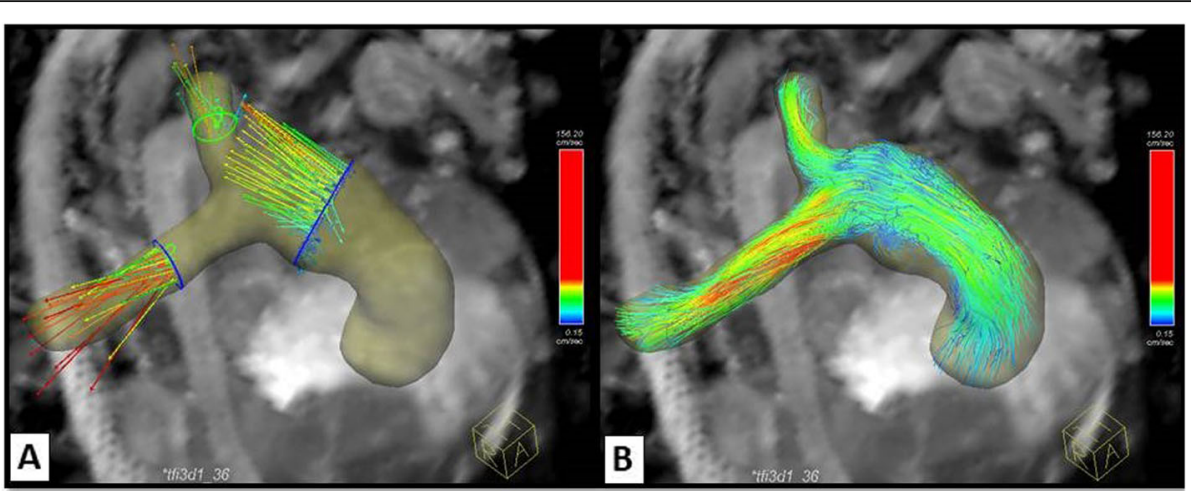

Fig. 3 4D-CMR flow of the MPA, RPA, and LPA of a surgically repaired TOF patient with RV-PA conduit. a Vector field maps showing the velocity and direction of blood flow across pulmonary arteries during systole (arrows). This type of visualization provides a quick overview of velocity fields. $\mathbf{b}$ Streamlines color map showing also the forward flow across pulmonary arteries during systole. This type of visualization is useful to visualize 3D velocity fields at discrete time points. A high-systolic velocity across the RPA is noted by the two methods (red color map). CMR: cardiac magnetic resonance, MPA: main pulmonary artery, RPA: right pulmonary artery, LPA: left pulmonary artery, TOF: tetralogy of Fallot

years. The RV EF was $56.3 \pm 8.6 \%$, RV EDVI $104 \pm 23.5$ $\mathrm{ml} / \mathrm{m}^{2}$, RV ESVI $45 \pm 15.9 \mathrm{ml} / \mathrm{m}^{2}$, and RV SVI $58.5 \pm$ $15.7 \mathrm{ml} / \mathrm{m}^{2}$.

The SVI across the MPA, RPA, and LPA were nearly similar between 4D-CMR and 2D-PC flows with no statistically significant differences $(44.87 \pm 35.71$ vs. $48.11 \pm$
$41.79 \mathrm{ml} / \mathrm{m}^{2}, 27.84 \pm 17.11$ vs. $30.46 \pm 20.10 \mathrm{ml} / \mathrm{m}^{2}$, and $21.13 \pm 17.11$ vs. $22.92 \pm 20.26 \mathrm{ml} / \mathrm{m}^{2}, P=0.179,0.218$, and 0.091 respectively) (Table 1 ).

Regarding the RF across the MPA, RPA, and LPA, it was significantly higher by $4 \mathrm{D}-\mathrm{CMR}$ compared to 2DPC flow with statistically significant differences $(24.25 \pm$

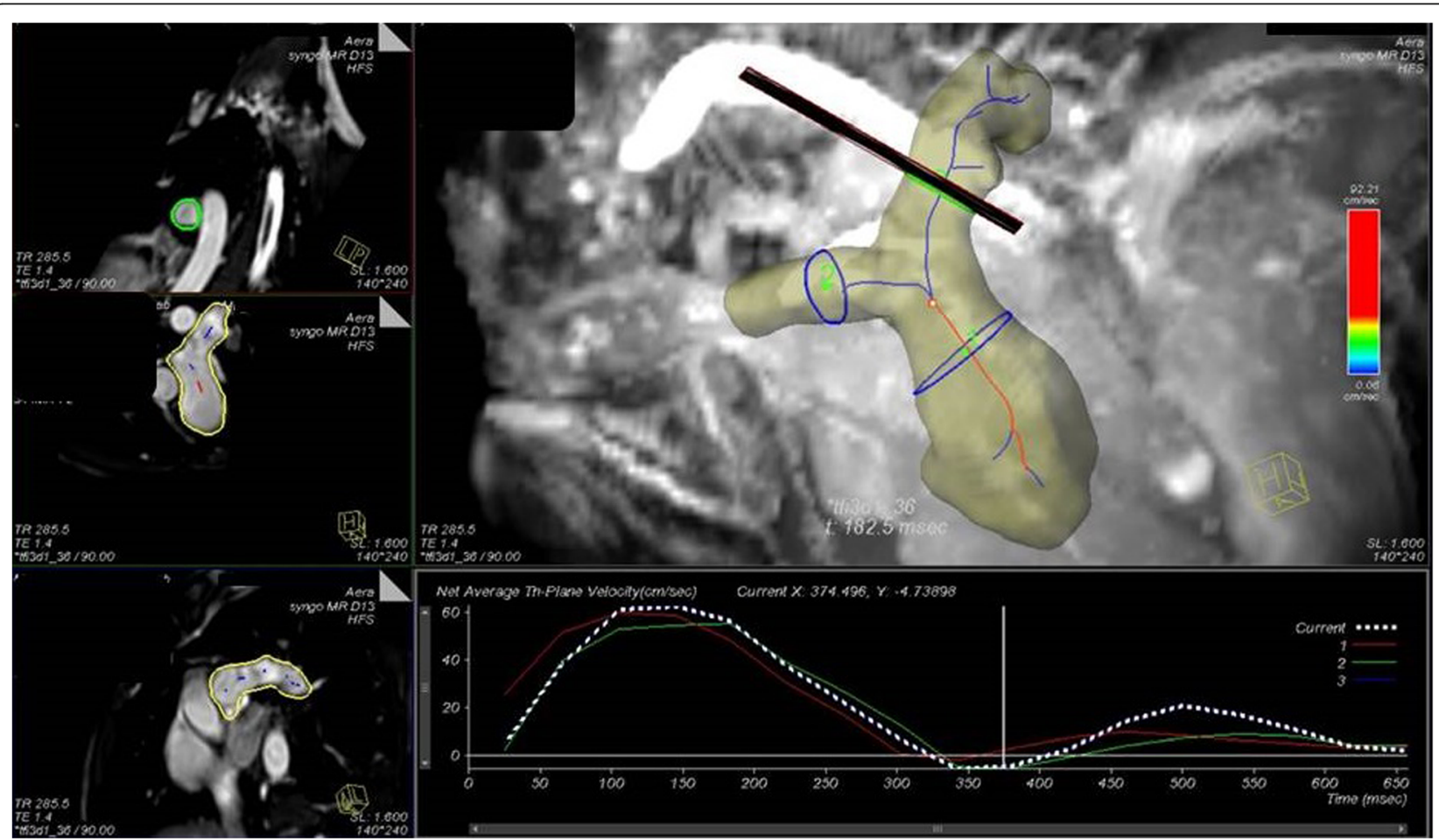

Fig. 4 4D-CMR flow of the MPA, RPA, and LPA in a surgically repaired TOF patient with RVOT patch showing the offline planning of areas of interest at the proximal segments of these vessels to acquire all flow data and flow-time curves showed. CMR: cardiac magnetic resonance, MPA: main pulmonary artery, RPA: right pulmonary artery, LPA: left pulmonary artery 
22.27 vs. $20.53 \pm 18.98 \%, 18.75 \pm 19.69$ vs. $14.29 \pm$ $16.57 \%$, and $26.36 \pm 22.17$ vs. $21.51 \pm 18.95 \%, P=$ $0.027,0.039$, and 0.046 , respectively) (Table 1 ).

Regarding the PSV across the MPA, RPA, and LPA, it was significantly higher by $4 \mathrm{D}-\mathrm{CMR}$ compared to $2 \mathrm{D}$ PC flow with statistically significant differences (158.15 \pm 28.78 vs. $148.34 \pm 24.65 \mathrm{~cm} / \mathrm{s}, 160.09 \pm 46.33$ vs. $144.50 \pm 46.98 \mathrm{~cm} / \mathrm{s}$, and $154.30 \pm 40.62$ vs. $136.36 \pm$ $39.79 \mathrm{~cm} / \mathrm{s}, P=0.003,<0.001$, and 0.002 , respectively) (Table 1).

Bland-Altman plots were used to correlate between the flow data obtained by $4 \mathrm{D}-\mathrm{CMR}$ and $2 \mathrm{D}-\mathrm{PC}$ flows and showed a good agreement between the two studied methods regarding the SVI, RF, and PSV across the MPA, RPA, and LPA as indicated by the data point distribution. (Fig. 5)

\section{Discussion}

This study was conducted to assess the flow pattern across the pulmonary arteries in surgically corrected TOF patients by 4D-CMR flow and we compared it with the conventional 2D-PC flow.

The feasibility of 4D-CMR flow for the comprehensive assessment of flow patterns in pulmonary arteries and pulmonary hemodynamics was successfully demonstrated in this group of patients with surgically repaired TOF.

In our study, there was no significant difference in the SVI across the pulmonary arteries between 4D-CMR and 2D-PC flow while the RF was significantly higher by 4D-CMR flow in comparison to 2D-PC flow. However, there was a strong positive correlation between both

Table 1 Comparison between the 4D-CMR and 2D-PC flows across the pulmonary arteries regarding the stroke volume index, regurgitation fraction, and peak systolic velocity

\begin{tabular}{|c|c|c|c|}
\hline & 4D-CMR flow & 2D-PC flow & $\begin{array}{l}\text { Significance } \\
\text { paired } t \text { test }\end{array}$ \\
\hline \multicolumn{4}{|l|}{$M P A$} \\
\hline $\mathrm{SVI}\left(\mathrm{ml} / \mathrm{m}^{2}\right)$ & $44.87 \pm 35.71$ & $48.11 \pm 41.79$ & $P=0.179$ \\
\hline RF $(\%)$ & $24.25 \pm 22.27$ & $20.53 \pm 18.98$ & $P=0.027^{*}$ \\
\hline PSV $(\mathrm{cm} / \mathrm{s})$ & $158.15 \pm 28.78$ & $148.34 \pm 24.65$ & $P=0.003^{*}$ \\
\hline \multicolumn{4}{|l|}{$R P A$} \\
\hline $\mathrm{SVI}\left(\mathrm{ml} / \mathrm{m}^{2}\right)$ & $27.84 \pm 17.11$ & $30.46 \pm 20.10$ & $P=0.218$ \\
\hline RF (\%) & $18.75 \pm 19.69$ & $14.29 \pm 16.57$ & $P=0.039^{*}$ \\
\hline PSV $(\mathrm{cm} / \mathrm{s})$ & $160.09 \pm 46.33$ & $144.50 \pm 46.98$ & $P<0.001^{*}$ \\
\hline \multicolumn{4}{|l|}{$\angle P A$} \\
\hline $\mathrm{SVI}\left(\mathrm{ml} / \mathrm{m}^{2}\right)$ & $21.13 \pm 17.11$ & $22.92 \pm 20.26$ & $P=0.091$ \\
\hline RF (\%) & $26.36 \pm 22.17$ & $21.51 \pm 18.95$ & $P=0.046^{*}$ \\
\hline PSV $(\mathrm{cm} / \mathrm{s})$ & $154.30 \pm 40.62$ & $136.36 \pm 39.79$ & $P=0.002^{*}$ \\
\hline
\end{tabular}

$P P$ value for Wilcoxon signed ranks test for comparing between 4D-CMR and 2D-PC flows

*Statistically significant at $P \leq 0.05$ methods regarding the SVI and RF across the pulmonary arteries.

Similar to our study, Jacobs et al. found a good agreement between 4D-CMR and 2D-CMR flows regarding the RF in pulmonary arteries [12]. Also, Raluca et al. [13] demonstrated a good correlation between both methods regarding flow volumes.

On the other side, Isorni et al. [14] found that 4D-CMR flow underestimated the pulmonary RF in comparison to 2D-PC flow in a study performed on 18 surgically repaired TOF patients. However, there was a good agreement between both methods.

Regarding the PSV across the pulmonary arteries, it was significantly higher by 4D-CMR flow in comparison to $2 \mathrm{D}-\mathrm{PC}$ flow, yet with a strong positive correlation between both methods. Similarly, in a previous study performed by Nordmeyer et al. [15] which was conducted on 18 patients with semilunar valves stenosis, they reported higher PSV with 4D-CMR flow compared to 2DPC flow when they were measured at the level with the highest velocity within the vessel and was more comparable to echocardiography [15].

Moreover, in another study performed by Gabbour et al. [16], they reported that 2D-PC flow significantly underestimated the PSV across MPA compared to echocardiography, while 4D-CMR was highly comparable to results obtained by echocardiography [16].

In the contrary, Raluca et al. [13] found that 4D-CMR flow underestimated the PSV when they performed the measurements at the same level as when using 2D-PC flow and they attributed this to the lower temporal resolution of 4D-CMR flow [13].

Finally, the major advantage of 4D-CMR flow which makes it superior to the conventional 2D-PC flow is that it acquires the entire volume in a single free-breathing acquisition. Additionally, flow can be measured anywhere and in any direction within the vessel of interest by prescribing infinite number of planes during offline analysis and the acquired data can be visualized for anatomy, flow velocity, volumes, and direction. In the contrary, 2D-PC flow requires separate acquisition of each vessel of interest with the probability of repeating it many times to enhance the plane in such complex CHD, further prolonging the scan time. In our study, the entire volume acquisition of 4D-CMR flow took on average 10 min while 2D-PC flow acquisition took much longer time for acquiring and optimizing the planes of interest within MPA, RPA, and LPA. Saving scan time is of extreme importance in the assessment of patients with complex CHD especially in the pediatric group.

\section{Study limitations}

This study is limited by the relatively small number of patients. Larger patient cohorts and further follow-up 


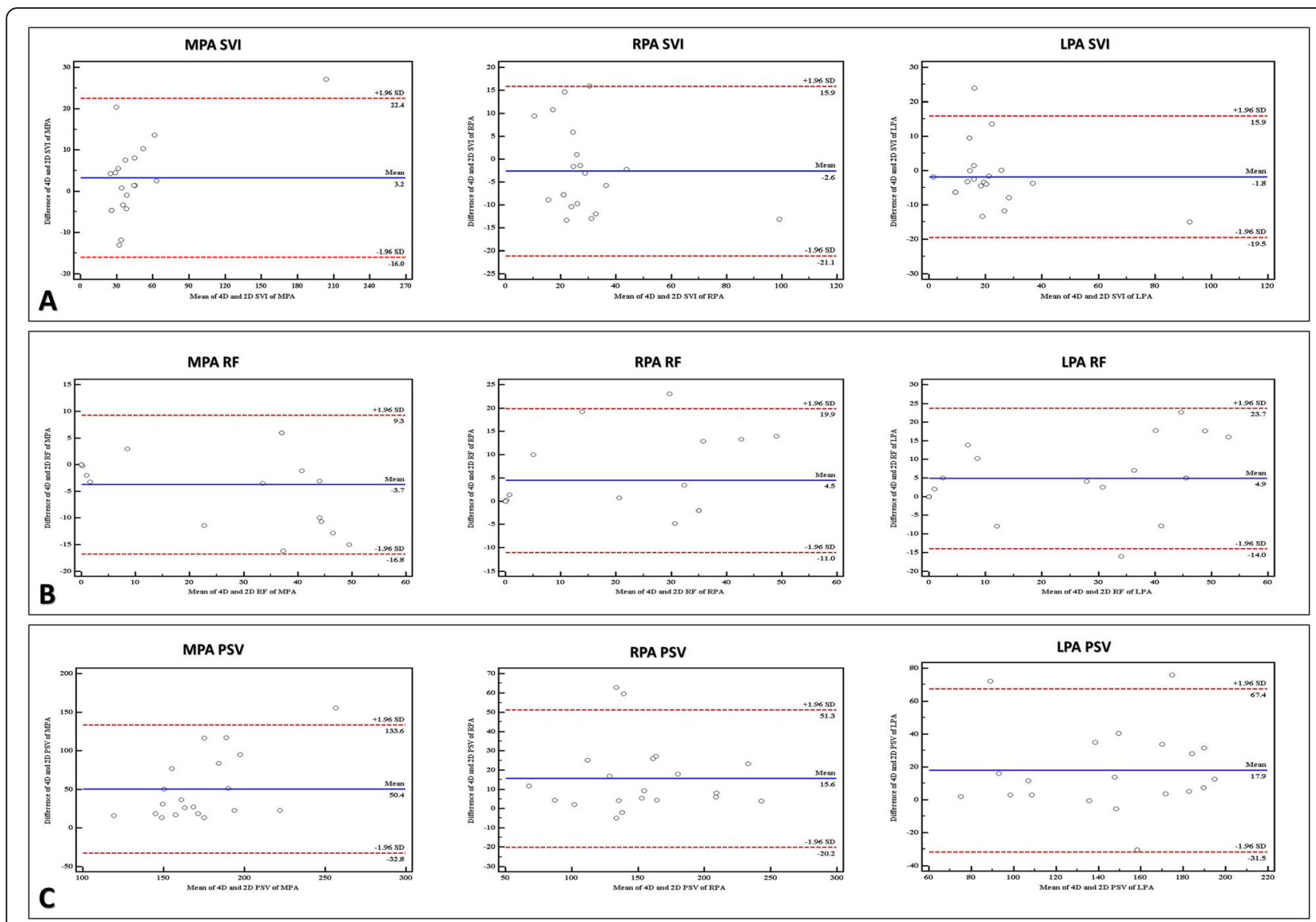

Fig. 5 Bland-Altman plots comparing between 4D-CMR and 2D-PC flow across the pulmonary arteries. The middle line represents the mean difference while the upper and lower lines represent the \pm 1.96 standard deviation limit. The plots show the lines of equality between and $95 \%$ limits of agreement between the two studied methods for the SVI (a), RF (b), and PSV (c) across the pulmonary arteries. SV: stroke volume, RF: regurgitation fraction, PSV: peak systolic velocity, MPA: main pulmonary artery, RPA: right pulmonary artery, LPA: left pulmonary artery

examinations are essential to learn more about altered pulmonary hemodynamics following TOF repair.

Another limitation of this study is the absence of a gold standard for pulmonary flow analysis to be correlated with the two studied methods.

\section{Conclusion}

There was a good agreement between 4D-CMR and 2DPC flow regarding pulmonary flow measurements. Because of its major advantage of performing a comprehensive flow assessment in a shorter time, 4D-CMR flow plays an important role in the assessment of patients with complex CHD especially in the pediatric group. Further studies on larger patient cohort are needed to determine its added value in the assessment of rightsided hemodynamics.

\section{Abbreviations}

2D: Two dimensional; 3D: Three dimensional; 4D: Four dimensional; CHD: Congenital heart disease; CMR: Cardiovascular magnetic resonance; ED: End-diastolic; ES: End-systolic; LPA: Left pulmonary artery; LV: Left ventricle; MPA: Main pulmonary artery; PA: Pulmonary artery; PC: Phase contrast; PSV: Peak systolic velocity; RF: Regurgitation fraction; RPA: Right pulmonary artery; PR: Pulmonary regurgitation; RV: Right ventricle; RVOT: Right ventricle outflow tract; SSFP: Steady state free precession; SV: Stroke volume; TOF: Tetralogy of Fallot; VSD: Ventricular septal defect

\section{Acknowledgements}

The authors acknowledge Dr. Soha Romeih, lecturer of cardiology, Tanta University, and the director of cardiovascular imaging unit at Aswan Heart Centre for her continuous support to complete this work.

\section{Consent of publication}

Not applicable.

\section{Authors' contributions}

M.N.S. has contributed to the work by scanning all included patients on our 1.5T CMR scanner and performed the post-processing analysis for all patients. R.E. revised the study design and performed the statistical analysis. M.S. and A.A. drafted the manuscript and M.E. revised the work. All authors have approved the submitted manuscript.

\section{Funding}

There was no source of funding for this study.

Availability of data and materials

The datasets used during the current study are available from the corresponding author on reasonable request. 


\section{Ethics approval and consent to participate}

An informed written consent was taken from each participant's father or mother. This study was approved by Research Ethics Committee, Quality Assurance Unit, Faculty of Medicine, Tanta University, with the following approval code: 31542/05/17.

\section{Competing interests}

The authors declare that they have no competing interests.

Received: 20 May 2020 Accepted: 27 August 2020

Published online: 07 September 2020

\section{References}

1. Apitz C, Webb GD, Redington AN (2009) Tetralogy of fallot. Lancet. 374(9699):1462-1471

2. Lillehei CW, Varco RL, Cohen M, Warden HE, Patton C, Moller JH (1986) The first open-heart repairs of ventricular septal defect, atrioventricular communis, and tetralogy of Fallot using extracorporeal circulation by crosscirculation: a 30-year follow-up. Ann Thorac Surg 41(1):4-21

3. Huehnergarth KV, Gurvitz M, Stout KK, Otto CM (2008) Repaired tetralogy of Fallot in the adult: monitoring and management. Heart. 94(12):1663-1669

4. Davlouros PA, Kilner PJ, Hornung TS, Li W, Francis JM, Moon JCC et al (2002) Right ventricular function in adults with repaired tetralogy of Fallot assessed with cardiovascular magnetic resonance imaging: detrimental role of right ventricular outflow aneurysms or akinesia and adverse right-to-left ventricular interaction. J Am Coll Cardiol 40(11):2044-2052

5. Kilner PJ, Geva T, Kaemmerer H, Trindade PT, Schwitter J, Webb GD (2010) Recommendations for cardiovascular magnetic resonance in adults with congenital heart disease from the respective working groups of the European Society of Cardiology. Eur Heart J 31(7):794-805. Available from: https://doi.org/10.1093/eurheartj/ehp586

6. Norton Kl, Tong C, Glass RBJ, Nielsen JC (2006) Cardiac MR imaging assessment following tetralogy of Fallot repair 1. Radiographics. 26(1):197-211

7. Markl M, Harloff A, Bley TA, Zaitsev M, Jung B, Weigang E et al (2007) Timeresolved $3 D \mathrm{MR}$ velocity mapping at $3 \mathrm{~T}$ : improved navigator-gated assessment of vascular anatomy and blood flow. J Magn Reson Imaging 25(4):824-831

8. Bächler P, Valverde I, Pinochet N, Nordmeyer S, Kuehne T, Crelier G et al (2013) Caval blood flow distribution in patients with Fontan circulation: quantification by using particle traces from 4D flow MR imaging. Radiology. 267(1):67-75

9. Geiger J, Hirtler D, Bürk J, Stiller B, Arnold R, Jung B et al (2014) Postoperative pulmonary and aortic 3D haemodynamics in patients after repair of transposition of the great arteries. Eur Radiol 24(1):200-208

10. Hirtler D, Geiger J, Jung B, Markl M, Arnold R (2013) 4-D MRI flow analysis in the course of interrupted aortic arch reveals complex morphology and quantifies amount of collateral blood flow. Pediatr Radiol 43(8):1037-1040

11. Muñoz DR, Markl M, JLM M, Barker A, Fernández-Golfín C, Lancellotti P et al (2013) Intracardiac flow visualization: current status and future directions. Eur Heart J Cardiovasc Imaging jet086

12. Jacobs KG, Chan FP, Cheng JY, Vasanawala SS, Maskatia SA (2020) 4D flow vs. $2 \mathrm{D}$ cardiac MRI for the evaluation of pulmonary regurgitation and ventricular volume in repaired tetralogy of Fallot: a retrospective case control study. Int J Card Imaging:1-13

13. Chelu RG, Wanambiro KW, Hsiao A, Swart LE, Voogd T, van den Hoven AT et al (2016) Cloud-processed 4D CMR flow imaging for pulmonary flow quantification. Eur J Radiol 85(10):1849-1856

14. Isorni M-A, Tortigue M, Ben Moussa N, Hascoët S, Monnot S (2018) 4D Flow CMR analysis in repaired Tetralogy of Fallot: Where we are and where we are going. Arch Cardiovasc Dis Suppl 10(3):285 Available from: http://www. sciencedirect.com/science/article/pii/S1878648018302192

15. Nordmeyer S, Riesenkampff E, Messroghli D, Kropf S, Nordmeyer J, Berger F et al (2013) Four-dimensional velocity-encoded magnetic resonance imaging improves blood flow quantification in patients with complex accelerated flow. J Magn Reson Imaging 37(1):208-216

16. Gabbour M, Schnell S, Jarvis K, Robinson JD, Markl M, Rigsby CK (2015) 4-D flow magnetic resonance imaging: blood flow quantification compared to 2-D phase-contrast magnetic resonance imaging and Doppler echocardiography. Pediatr Radiol 45(6):804-813. Available from. https://doi. org/10.1007/s00247-014-3246-z

\section{Publisher's Note}

Springer Nature remains neutral with regard to jurisdictional claims in published maps and institutional affiliations.

\section{Submit your manuscript to a SpringerOpen ${ }^{\circ}$ journal and benefit from:}

- Convenient online submission

- Rigorous peer review

- Open access: articles freely available online

- High visibility within the field

- Retaining the copyright to your article

Submit your next manuscript at $\boldsymbol{\nabla}$ springeropen.com 